\title{
Epidermal Growth Factor Inhibitor-Induced Cutaneous Toxicity Im- proves with Moisturizers
}

\author{
Ichiko Morino $^{1}$, Aika Okuno ${ }^{2}$, Yuka Hirakawa ${ }^{2}$, Yumiko Saya ${ }^{1 *}$, Yumi Murakami ${ }^{1}$, Fukumi Furukawa $^{2}$, \\ Hiroshi Matsunaka ${ }^{1}$
}

${ }^{1}$ NOV Academic Research, TOKIWA Pharmaceutical Co., Ltd. E-mail: yumiko_saya@n1.noevir.co.jp

${ }^{2}$ Department of Dermatology, Takatsuki Red Cross Hospital

\begin{abstract}
Although epidermal growth factor receptor (EGFR) inhibitors are one of the most effective treatment options for lung cancer, they frequently cause cutaneous toxicity that can lead to treatment discontinuation. Dryness, which is a common form of cutaneous toxicity, is usually treated using medical moisturizing agents. We aimed to investigate the treatment of cutaneous toxicity caused by EGFR inhibitors by comparing patients who used a cosmetic moisturizer with those who used conventional medical moisturizers. This study included 12 patients with lung cancer, who were receiving EGFR inhibitors and using topical medical moisturizers. The participants were assigned to a group that continued using medical moisturizers or a group that began using $\mathrm{NOV}^{\circledR}$ skin cream D. The study's findings showed that like conventional medical moisturizers, $\mathrm{NOV}^{\circledR}$ skin cream D improved the cutaneous dryness caused by EGFR inhibitors and that it might additionally improve patients' quality of life. Also, we obtained novel findings that NOV ${ }^{\circledR}$ skin cream D normalized keratinization, which is a component of normal skin cell differentiation impeded by EGFR inhibitors. Hence, the cosmetic moisturizer may help to prevent the discontinuation of EGFR inhibitors, thereby ensuring their continuous therapeutic effects.
\end{abstract}

Keywords: Cutaneous Toxicity; Epidermal Growth Factor Receptor Inhibitors; Moisturizer; Quality of Life; Stratum Corneum Water Content

\section{Introduction}

Epidermal growth factor receptor (EGFR) inhibitors, which target EGFR mutations, are used to treat lung cancer $^{[1]}$. However, cutaneous toxicities, including dry skin, acne-like eruptions, and rashes, occur frequently ${ }^{[2]}$, and the quality of life (QOL) of the patients declines. Therefore, administering EGFR inhibitors continuously is difficult ${ }^{[3]}$. Given that side-effects indicate cancer cell sensitivity ${ }^{[4]}$ and they correlate with the therapeutic effect, it is important to continue the administration of EGFR inhibitors while managing their side-effects.

Of the cutaneous toxicities, which are a major side-effect of EGFR inhibitors, dryness can be alleviated or prevented by using moisturizers. Medical moisturizers, including preparations that contain heparin-like compounds, are widely used ${ }^{[5-7]}$, but many patients complain about the sticky feeling associated with using these medical moisturizers, and if the use of moisturizers is to continue, moisturizers that match patients' preferences are required. This study aimed to investigate the treatment of cutaneous toxicities caused by EGFR inhibitors by comparing patients who used a cosmetic moisturizer, namely, $\mathrm{NOV}^{\circledR}$ skin cream D (Noevir Co., Ltd., Kobe, Japan), with those who used conventional medical moisturizers.

\section{Materials and methods}

\subsection{Participants}

The participants were patients with EGFR mutation-positive lung cancer who visited Takatsuki Red Cross Hospital Department of Dermatology between January and October 2018. These patients were adminis-

Copyright (C) 2020 Ichiko Morino et al.

doi: 10.24294/ti.v4.i2.1187

EnPress Publisher LLC.This work is licensed under the Creative Commons Attribution-NonCommercial 4.0 International License (CC BY-NC 4.0).

http://creativecommons.org/licenses/ by/4.0/ 
tered afatinib $\left(\right.$ Giotrif $\left.^{\circledR}\right)$, gefitinib (Iressa $\left.{ }^{\circledR}\right)$, or osimertinib (Tagrisso ${ }^{\circledR}$ ), and they applied moisturizers, including $\mathrm{NOV}^{\circledR}$ skin cream D, Hirudoid ${ }^{\circledR}$ soft ointment $(0.3 \%)$ (Maruho Co., Ltd., Osaka, Japan), or Besoften ${ }^{\circledR}$ Lotion (0.3\%) (Teikoku Seiyaku Co. Ltd., Kagawa, Japan), externally to dry skin to counter the drugs' side-effects.

\subsection{Moisturizer application}

The patients were randomly assigned to two groups, of which, one continued using the medical moisturizers (Medical moisturizer group) and the other started using the cosmetic moisturizing cream (Moisturizing cream group). The participants applied the moisturizers (1-2 $\mathrm{mg} / \mathrm{cm}^{2}$ ) systematically at home in the morning and evening, or after bathing for 8 weeks. The patients did not apply the moisturizers to the upper back region, which comprised the control region.

\subsection{Observation and assessments}

The patients' inner forearms, which received the moisturizers (application area), and their upper backs (control area) were evaluated.

\subsubsection{Evaluation of skin condition}

The levels of dryness, itchiness, and erythema on the left and right inner forearms and upper back were scored using a 5-grade scale, as follows: none: 0; slight: 1; mild: 2 ; moderate: 3 ; or severe: 4 , and the total scores were calculated.

\subsubsection{Stratum corneum analysis}

The stratum corneum water content was evaluated using a 3.5-MHz high-frequency conductance instrument (Skicon ${ }^{\circledR}-200 E X$; Yayoi Co., Ltd., Tokyo, Japan). Areas $(24 \mathrm{~mm} \times 50 \mathrm{~mm})$ of the stratum corneum on the right inner forearm and upper back were sampled using tape stripping (Cellotape ${ }^{\circledR}$; Nichiban Co., Ltd., Tokyo, Japan). The interleukin (IL)-1 receptor antagonist (IL-1ra) and IL- $1 \alpha$ levels were quantified using an enzyme-linked immunosorbent assay ${ }^{[8]}$, and the IL-1ra to IL- $1 \alpha$ ratios were calculated. The stratum corneum samples were stained immunohistologically for thymic stromal lymphopoietin (TSLP) ${ }^{[9]}$, and the fluorescence intensities per cell area were calculated. After staining the samples with gentian violet and brilliant green, the captured images were binarized, and the multilayer exfoliated cell area to the total cell area ratios were calculated to determine the degree of multilayer exfoliation. The levels of trypsin activity were then measured ${ }^{[10]}$.

\subsubsection{Patient surveys}

The patients' QOL was evaluated using the Skindex-16 questionnaire. Furthermore, a questionnaire was administered to the patients to assess the ease of application and the moist feelings associated with the using of the moisturizers.

\subsection{Statistical analyses}

The Wilcoxon signed-rank test was used to analyze the skin condition's scores, the water content of the stratum corneum, IL-1ra to IL- $1 \alpha$ ratios, TSLP levels, degrees of multilayer exfoliation, trypsin activity, and the QOL scores on weeks 0-8. The Mann-Whitney U test was used to compare data from the inner forearm (application area) and the upper back (control area) regions, and the Medical moisturizer and the Moisturizing cream groups. The groups' questionnaires were analyzed using the $\chi^{2}$-test. The statistical analyses were performed using SPSS $^{\circledR} 21.0$ (SPSS Inc., Chicago, IL, USA), and a value of $p<0.1$ was considered significant.

\subsection{Ethics statement}

The Takatsuki Red Cross Hospital Ethics Committee approved this study (H29-20). All study participants provided informed consent.

\section{Results}

\subsection{Background of participants}

The participants comprised one man and 11 women whose mean age was $72.8 \pm 8.3$ years ( $58-85$ years). The groups did not differ regarding sex and age. All of the patients used the moisturizers continuously without any adverse events. The skin symptoms did not worsen in either group.

\subsection{Evaluation of skin condition}

Figure 1 shows the total dryness, itching, and erythema scores from the patients' inner forearms and upper backs. In both groups, the inner forearms' total scores had decreased significantly by week 8 compared with those at week 0 . At week 8 , the inner forearm's total score was significantly lower than the upper back's total score in the Moisturizing cream group.

\subsection{Stratum corneum analyses}

Table 1 shows the analyses of the stratum corneum. Compared with week 0 , the water content of the upper back's stratum corneum was significantly lower at week 8 in the Medical moisturizer group. In both groups, 
the TSLP levels in the stratum corneum of the inner forearm and upper back were significantly higher at week 8 compared with those at week 0 . In the Moisturizing cream group, the degree of multilayer exfoliation of the inner forearm was significantly lower at week 8 compared with that at week 0 . In the Moisturizing cream group, the degree of multilayer exfoliation of the inner forearm was significantly lower compared with that of the upper back at week 8. In the Moisturizing cream group, the degree of multilayer exfoliation of the inner forearm was significantly lower than that in the Medical moisturizing group at week 8 .

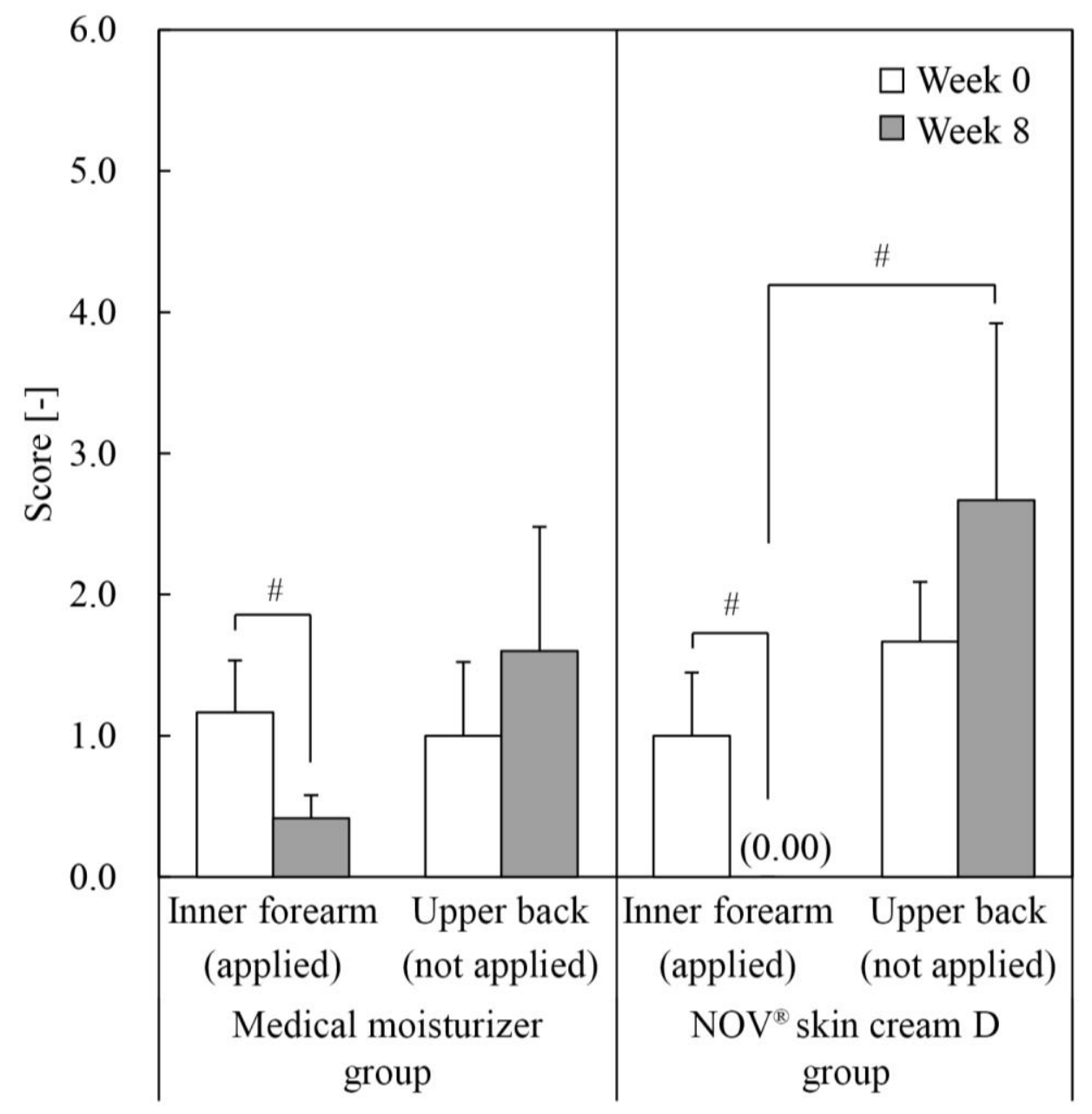

Skin finding scores: Total score for dryness, itching, and erythema

Mean \pm S.E., medical moisturizer group (inner forearm, $n=6$; upper back, $n=5$ ), $\mathrm{NOV}^{\circledR}$ skin cream $\mathrm{D}$ group $(n=6)$,

Week 0 vs. Week 8: Wilcoxon signed-rank test inner forearm (applied) vs. upper back (not applied) and medical moisturizer group vs. $\mathrm{NOV}^{\circledR}$ skin cream D group: Mann-Whitney U test, ${ }^{\#}: p<0.1$

Figure 1. Skin finding scores. 


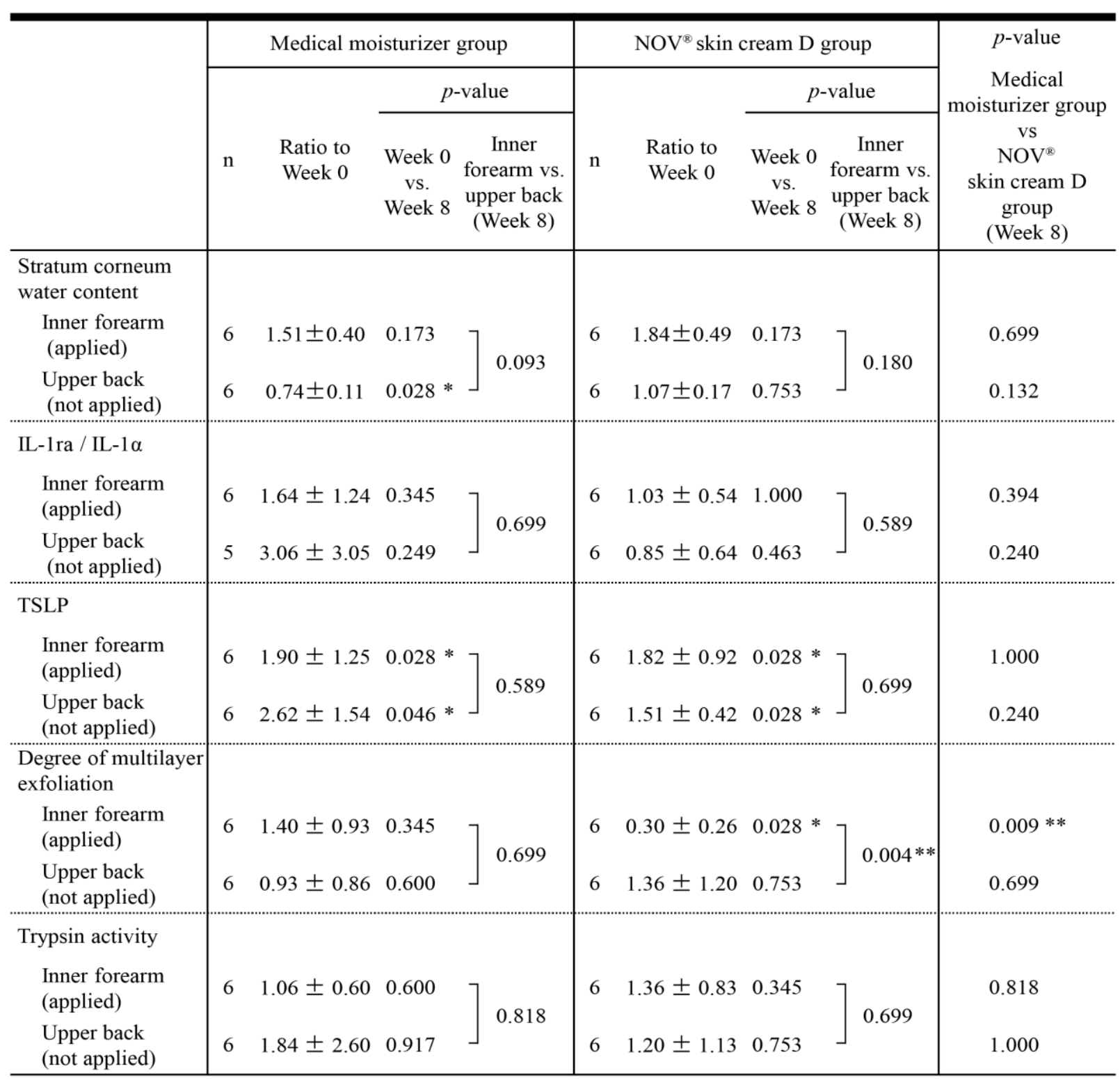

Table 1. Stratum corneum analysis

Ratio to Week 0: Ratio in Week 8 compared with Week 0 as 1, values represented as mean \pm S.D., Week 0 vs. Week 8: Wilcoxon signed-rank test inner forearm (applied) vs. upper back (not applied) and medical moisturizer group vs. NOV ${ }^{\circledR}$ skin cream D group: Mann-Whitney U test, * : $p<0.05, * *: p<0.01$

TSLP: thymic stromal lymphopoietin

\subsection{Patient survey findings}

Figure 2 shows the results from the QOL evaluations using the Skindex-16 questionnaire. In the Moisturizing cream group, the patients' symptoms, emotions, and global scores were significantly lower at week 8 than those at week 0 . The symptom score was significantly lower in the Moisturizing cream group than that in the Medical moisturizing group at week 8 .
Regarding "ease of application", 50\% (3/6) of the patients in the Medical moisturizer group and 67\% (4/6) of the patients in the Moisturizing cream group indicated that the moisturizers were easy to apply. Regarding "moist feeling", 33\% (2/6) of the patients in the Medical moisturizer group and $67 \%(4 / 6)$ of the patients in the Moisturizing cream group indicated that the moisturizers were moisturizing. 


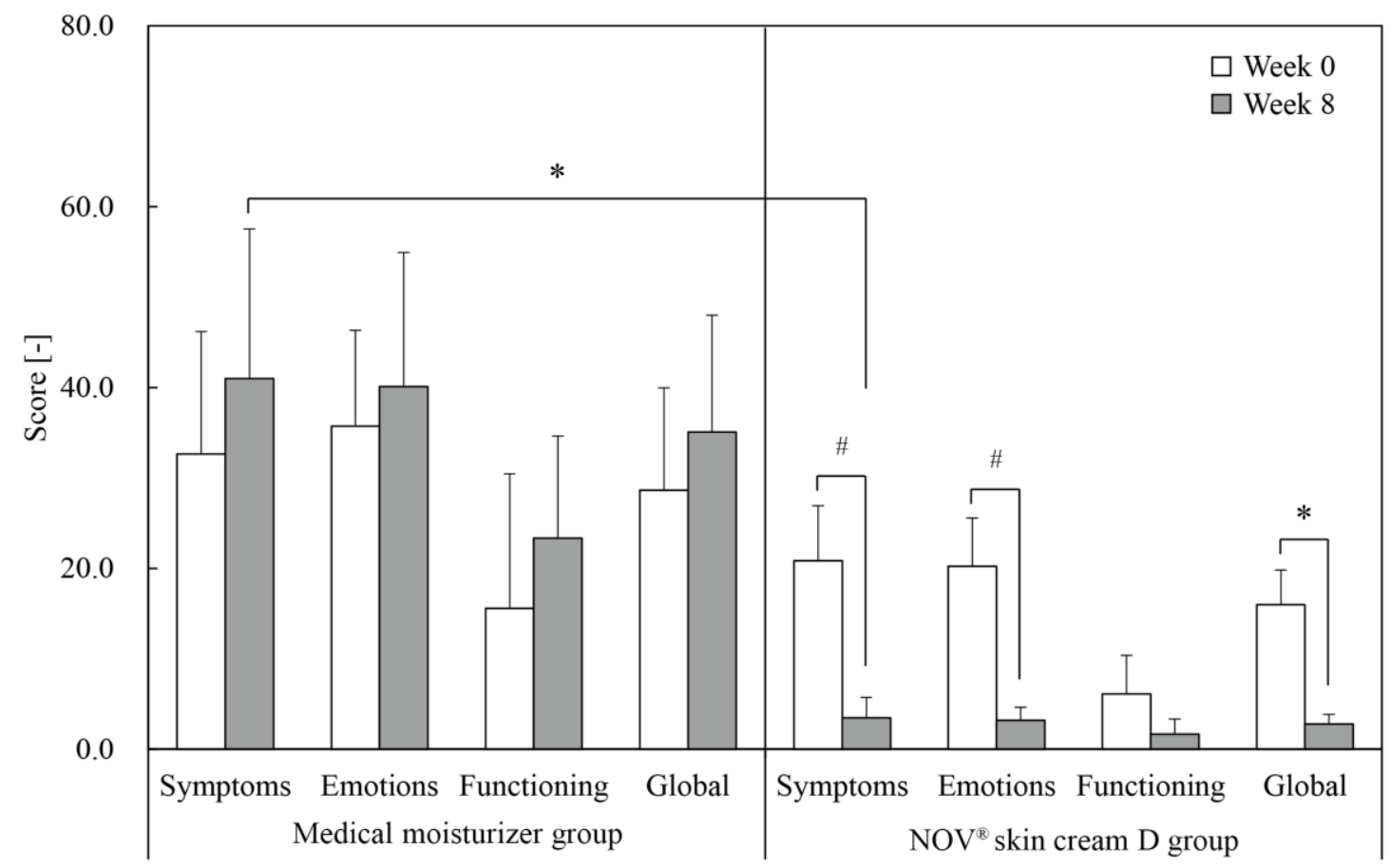

Mean \pm S.E., medical moisturizer group $(n=6), \mathrm{NOV}^{\circledR}$ skin cream $\mathrm{D}$ group $(\mathrm{n}=6)$, Week 0 vs. Week 8: Wilcoxon signed-rank test, medical moisturizer group vs. $\mathrm{NOV}^{\circledR}$ skin cream D group: Mann-Whitney $\mathrm{U}$ test, ${ }^{\#}: p<0.1, *: p<0.05$

\section{Discussion}

In this study, patients with lung cancer who were administered EGFR inhibitors were assigned to the Medical moisturizer group or the Moisturizing cream group, and the effectiveness of medical moisturizers and a moisturizing cream was compared for 8 weeks. None of the moisturizers were associated with any side-effects. After 8 weeks, the Moisturizing cream group had significantly lower degrees of multilayer exfoliation and lower symptom scores than the Medical moisturizing group. There was almost no difference in the other parameters investigated.

The results from the evaluation of the multilayer exfoliation suggested that the moisturizing creams normalized keratinization, which is a component of normal skin cell differentiation that is impeded by EGFR inhibitors ${ }^{[11]}$. Patients with atopic dermatitis have high TSLP levels in the stratum corneum ${ }^{[12]}$. The current study's results showed that TSLP production was similarly enhanced during the administration of EGFR inhibitors. EGFR inhibitors increase the levels of different chemokines ${ }^{[13]}$, but this is the first study of TSLP expression in the stratum corneum. The IL-1ra/IL-1 $\alpha$ ratio is an index of inflammation ${ }^{[8]}$, and trypsin is involved in stratum ssment based on Skindex-16. corneum exfoliation ${ }^{[10]}$. This is the first analysis of physiologically active substances in the stratum corneum following the administration of EGFR inhibitors. However, because of the small number of cases in this study, detailed analysis is limited. To elucidate the relationships among the physiologically active substances in the stratum corneum, the administration period, and skin symptoms, we intend to accrue a large number of patients. Compared with week 0, the symptom, emotion, and global scores were significantly lower in the Moisturizing cream group only at week 8 . Reducing a patient's QOL may hinder their on-going treatment with EGFR inhibitors ${ }^{[3]}$, and moisturizing creams may alleviate patients' psychological burdens.

\section{Conclusions}

The results from this study suggest that compared with conventional medical moisturizers, the moisturizing cream was superior at improving skin dryness associated with EGFR inhibitor administration. Furthermore, it may improve the QOL of patients. Regarding lung cancer treatment, applying the moisturizing cream should facilitate the continued administration of EGFR inhibitors and, thus, their therapeutic effects. 


\section{Conflicts of interest}

Tokiwa Pharmaceutical Co., Ltd. funded this clinical trial and the test products.

\section{Acknowledgments}

We would like to acknowledge Dr. Hideo Kita, director of the department of respiratory medicine at the Takatsuki Red Cross Hospital, for his cooperation in this clinical study.

\section{References}

1. Cufer T, Ovcaricek T, O'Brien ME. Systemic therapy of advanced non-small cell lung cancer: major-developments of the last 5-years. Eur J Cancer 2013; 49(6): 1216-1225. doi: 10.1016/j.ejca.2012. 11.021 .

2. Van Cutsem E. Challenges in the use of epidermal growth factor receptor inhibitors in colorectal cancer. Oncologist 2006; 11(9): 1010-1017. doi: 10.1634/theoncologist.11-9-1010.

3. Bianchini D, Jayanth A, Chua YJ, et al. Epidermal growth factor receptor inhibitor-related skin toxicity: Mechanisms, treatment, and its potential role as a predictive marker. Clin Colorectal Cancer 2008; 7(1): 33-43. doi: 10.3816/CCC.2008.n.005.

4. Orditura M, De Vita F, Galizia G, et al. Correlation between efficacy and skin rash occurrence following treatment with the epidermal growth factor receptor inhibitor cetuximab: A single institution retrospective analysis. Oncol Rep 2009; 21(4): 1023-1028. doi: 10.3892/or_00000319.

5. Lacouture ME, Mitchell EP, Piperdi B, et al. Skin toxicity evaluation protocol with panitumumab (STEPP), a phase II, open-label, randomized trial evaluating the impact of a pre-emptive skin treatment regimen on skin toxicities and quality of life in patients with metastatic colorectal cancer. J Clin Oncol 2010; 28(8): 1351-1357. doi: 10.1200/JCO. 2008.21.7828.

6. Nakahara T, Moroi Y, Takayama K, et al. Physiological change in cutaneous toxicity related to epidermal growth factor receptor (EGFR) inhibitors and the efficacy of emollient. Nishinihon J Dermatology 2014; 76: 242-247.

7. Nakahara T, Moroi Y, Takayama K, et al. Effective supportive care for patients with different physiological changes in various skin regions induced by EGFR inhibitors. Nishinihon J Dermatology 2015; 77 (4): 399-405. doi: 10.2336/ nishinihonhifu.77.399.

8. Terui T, Hirao T, Sato Y, et al. An increased ratio of interleukin-1 receptor antagonist to interleukin-1alpha in inflammatory skin diseases. Exp Dermatol 1998; 7(6): 327-334.

9. Fukushima S, Morita E, Tanioka M, et al. Clinical evaluation of moisturizers with physiological anal- ysis of stratum corneum TARC and TSLP. Journal of Cosmetics, Dermatological Sciences and Applications 2014; 4(1): 37-43. doi: 10.4236/ jcdsa.2014.41006.

10. Redoules D, Tarroux R, Assalit MF, et al. Characterisation and assay of five enzymatic activities in the stratum corneum using tape-strippings. Skin Pharmacol Appl Skin Physiol 1999; 12(4): 182-192. doi: 10.1159/000066242.

11. Lacouture ME. Mechanisms of cutaneous toxicities to EGFR inhibitors. Nat Rev Cancer 2006; 6(10): 803-812. doi: 10.1038/nrc1970.

12. Sano Y, Masuda K, Tamagawa-Mineoka $\mathrm{R}$, et al. Thymic stromal lymphopoietin expression is increased in the horny layer of patients with atopic dermatitis. Clin Exp Immunol 2013; 171(3): 330 337. doi: 10.1111/cei.12021.

13. Nakahara T. Epidermal growth factor inhibitor-induced skin toxicity: Clinical symptoms, management, and mechanisms. Nishinihon J Dermatology 2015; 77(3): 203-209. doi: 10.2336/ nishinihonhifu.77.203. 